\title{
Modelo matemático para predecir las propiedades físicas en madera de Eucalyptus nitens para diferentes alturas y edades del árbol
}

\author{
Mathematical model for predicting the physical properties in the wood of \\ Eucalyptus nitens for different heights and ages of tree
}

\section{Carlos Rozas ${ }^{\mathrm{I}}$, Claudio Montero ${ }^{\mathrm{II}}$}

\begin{abstract}
Resumen
El Eucalyptus nitens presenta gran potencial para utilizarse en productos de mayor valor agregado, debido principalmente a su tasa de crecimiento y adaptabilidad a diferentes suelos y climas. Sin embargo, su uso aún es limitado, debido al desconocimiento de sus propiedades físicas a lo largo del fuste. En este sentido, el objetivo de la investigación fue modelar sus propiedades físicas a diferente altura y edad del árbol. Para esto, se seleccionaron 40 árboles de 13 años, 12 de 15 años y 5 de 20 años, evaluando propiedades físicas desde la base $(0,3 \mathrm{~m})$ hasta el Diámetro Libre Utilizable de $8 \mathrm{~cm}$. Se determinaron la densidad básica y el contenido de humedad según norma NCh176/2 Of. 86 y NCh176/1 Of. 86 respectivamente. Los resultados mostraron una densidad básica a los 13 años de $474 \mathrm{~kg} / \mathrm{m}^{3}$, con un contenido de humedad de $116 \%$, a los 15 años fue de $496 \mathrm{~kg} / \mathrm{m}^{3} \mathrm{y} 108 \%$ y a los 20 años de $521 \mathrm{~kg} / \mathrm{m}^{3}$ y $104 \%$. Se observó un aumento del 4,6\% y $9,7 \%$ en la densidad básica a los 15 y 20 años con respecto a la observada a los 13 años. El modelo detectó una relación inversa entre la densidad básica y el contenido de humedad, presentando ambas una alta correlación con la altura y la edad del árbol. Finalmente, el modelo predijo con alto nivel de precisión las propiedades físicas en esta especie, por lo que puede usarse como una herramienta para apoyar en la toma de decisiones al momento de definir el uso de las trozas según el tipo de producto que se quiera obtener.

Palabras-clave: Densidad básica; Contenido de humedad; Análisis estadístico
\end{abstract}

\begin{abstract}
Eucalyptus nitens has a great potential for its use in higher value-added products, mainly due to its growth rate and adaptability to different soils and climates. However, its use is still limited, due to lack of knowledge of its physical properties throughout the log. In this sense, the objective of the research was to model the physical properties at different heights and age of the tree. To do so, 40 trees of 13 years, 12 of 15 years and 5 of 20 years were selected, evaluating their physical properties from the base $(0.3 \mathrm{~m})$ to the Usable Free Diameter of $8 \mathrm{~cm}$. The basic density and moisture content were determined by standard NCh176/2 Of. 86 and NCh176/1 Of. 86, respectively. The results showed a basic density at 13 years of 474 $\mathrm{kg} / \mathrm{m}^{3}$, with a moisture content of $116 \%$, at 15 years it was $496 \mathrm{~kg} / \mathrm{m}^{3}$ and $108 \%$ and at 20 years it was $521 \mathrm{~kg} /$ $\mathrm{m}^{3}$ and $104 \%$ respectively. There was an increase of $4.6 \%$ and $9.7 \%$ in the basic density at 15 and 20 years compared to 13 years. The model detected an inverse relationship between the basic density and moisture content, both presenting a high correlation with the height and age of tree. Finally, the model predicted with high level of precision the physical properties in Eucalyptus nitens trees. So, it can be used as a tool to support decision-making when defining the use of logs according to the type of product to obtain.
\end{abstract}

Keywords: Basic density; Moisture content; Statistical analysis

Ingeniero Maderas, Dr., Profesor Asociado, Departamento de Ingeniería en Maderas, Facultad de Ingeniería, Universidad del Bío-Bío, sede Concepción, Av. Collao 1202, C.P. 4081112, Concepción, Chile. erozas@ubiobio.cl (ORCID: 0000-0002-2104-1114)

Ingeniero Civil, Industrias de la Madera, MSc., Jefe de Laboratorio - Investigador, Laboratorio de Adhesivos y Materiales Compuestos, Universidad del Bío-Bío, sede Concepción, Av. Collao 1202, C.P. 4081112, Concepción, Chile. cmontero@ubiobio.cl (ORCID: 0000-0001-7176-1892) 


\section{Introducción}

En Chile existen 828.236 hectáreas de plantaciones de Eucalyptus, de las cuales un 31\% corresponde a Eucalyptus nitens (Deane \& Maiden) (INSTITUTO FORESTAL, 2016). Esta especie ha mostrando una tasa creciente de hectáreas plantadas, lo que ha generado un mayor esfuerzo por parte de investigadores e industria para obtener productos de alto valor agregado (NAVARRETE et al., 2008). Esto representa una gran oportunidad para el desarrollo y diversificación de la industria secundaria maderera en Chile (ROZAS et al., 2002). La principal ventaja competitiva del Eucalyptus nitens, radica en su adaptabilidad a climas fríos, una alta tasa de crecimiento, un interesante rendimiento volumétrico y corto periodo de rotación (INSTITUTO FORESTAL, 2010). Para aprovechar estas ventajas y con el fin de obtener productos de madera de alto valor agregado, se deben considerar sus propiedades físicas, las que influyen directamente en su comportamiento en servicio (WIMMER; DOWNES; EVANS, 2002; BURGOS, 2009). En este sentido, para Panshin y De Zeew (1980), Chafe (1985), Rozas et al. (2002), Wimmer, Downes y Evans (2002), Burgos (2009), la densidad de la madera es la propiedad de mayor relevancia, tanto por la simpleza de su obtención, como por su correlación matemática con otras propiedades de la madera. Visto desde esta perspectiva, se considera a la densidad básica como el parámetro de mayor importancia para caracterizar a la madera, lo que se ve reflejado en su amplio uso por parte de investigadores y en la industria (ROZAS et al., 2002; OMONTE; VALENZUELA, 2011). También se reconoce como un parámetro para definir la calidad, selección y clasificación en los procesos de conversión, de secado y de fabricación de papel (HILLIS; BROWN, 1984; CAMPOS et al.,1990).

Actualmente, es frecuente que la densidad de todo el árbol se determine mediante la extracción de una muestra retirada a la altura del DAP (diámetro de la altura de pecho), lo que corresponde aproximadamente a una altura de $1,3 \mathrm{~m}$, presentando este método adecuados resultados (BUSNARDO et al., 1987; BEETS et al., 2018). Sin embargo, la caracterización de la densidad básica en un rodal no es tarea sencilla, debido a que existe una variabilidad significativa entre especies, entre árboles y dentro del mismo árbol (ZOBEL; BUIJTENEN, 1989). Tal variación se debe a factores ambientales (ZOBEL; BUIJTENEN, 1989) y anatómicos como; el tipo de células y de su estructura, el diámetro de lumen, espesor de pared celular y la cantidad de extraíbles (PANSHIN; DE ZEEW, 1980; ZOBEL; BUIJTENEN, 1989).

En este sentido, el desarrollo de estrategias para una correcta utilización de la madera, con el propósito de obtener productos de mayor valor, requiere contar con información precisa de su densidad (KIMBERLEY et al., 2017; SEDLAR et al., 2019). Aspecto relevante al momento de caracterizar las propiedades del Eucalyptus nitens, para usarse como madera sólida (OMONTE et al., 2019). Estos esfuerzos han permitido desarrollar modelos para predecir la densidad radial y axial en la madera, en especies latifoliadas como Populus spp., Acacia spp. y Salix spp. (GUTIERREZ-VASQUEZ et al., 2012), así como para predecir las propiedades físicas en diferentes especies de Eucalyptus (BUSNARDO et al., 1987). También Omonte y Valenzuela (2011), buscaron relacionar el comportamiento de la densidad a diferentes alturas en árboles de Eucalyptus regnans. Algunos estudios realizados en árboles de Eucalyptus nitens, describieron la variación de la densidad básica con la altura. Entre estos trabajos, se encuentran Mariani et al. (2005), quienes realizaron mediciones desde la base hasta la zona apical, registrando valores de densidad básica entre $450 \mathrm{~kg} / \mathrm{m}^{3}$ y $527 \mathrm{~kg} / \mathrm{m}^{3}$, mostrando un incremento significativo a partir del segundo tercio de la altura total del árbol. Un estudio similar de Rozas y Lisperguer (2005), en árboles de Eucalyptus nitens de 11 años, evaluó la densidad básica entre los 0,3 m y los 30,3 m de la altura total del árbol, reportando valores entre $496 \mathrm{~kg} / \mathrm{m}^{3}$ y $598 \mathrm{~kg} / \mathrm{m}^{3}$.

En general, los esfuerzos por comprender los factores que impactan sobre la variación de la densidad básica en el Eucalyptus nitens, aún son escasos y acotados, lo cual limita una adecuada utilización de esta especie. En este sentido, es relevante ampliar y comprender los factores que influyen sobre la densidad y el contenido de humedad, en donde es relevante considerar

Ci. Fl., Santa Maria, v. 30, n. 4, p. 980-994, out./dez. 2020 
aspectos como la homogeneidad y magnitud de estas propiedades con la altura y edad del árbol, lo que también permite su procesamiento de forma eficiente (TOMAZELLO, 1985; QUILHÓ; MIRANDA; PEREIRA, 2006; OMONTE; VALENZUELA, 2011; GUTIERREZ-VASQUEZ et al., 2012). Sobre la base a estos antecedentes, se definió como objetivo de esta investigación, predecir mediante modelos matemáticos la variación de la densidad básica y el contenido de humedad a diferente edad y altura del árbol. Con el fin de proponer una herramienta que permita apoyar la toma de decisiones para definir la utilización de las trozas de Eucalyptus nitens basándose en estas propiedades. Siendo este aspecto relevante, para obtener productos con mayor valor agregado, lo cual finalmente permitiría incrementar la participación en el mercado de los productos obtenidos de esta especie.

\section{Materiales y métodos}

\section{Procedencia de los árboles seleccionados}

Se utilizaron árboles de Eucalyptus nitens pertenecientes a la empresa Forestal Natalhue Ltda., localizada en la provincia de Valdivia, específicamente en el fundo Huenuye norte, sector Lanco-Panguipulli $\left(39^{\circ} 29^{\prime} 78^{\prime \prime S}\right.$ y $\left.78^{\circ} 41^{\prime} 34^{\prime \prime O}\right)$. Según la clasificación de Köppen, esta zona presenta un clima oceánico de corta sequía estival (Cfsb) con una precipitación de $2.351 \mathrm{~mm} / \mathrm{año}$, que se caracteriza por una alta humedad relativa y pluviosidad, así como por bajas temperaturas (DIRECCIÓN METEOROLÓGICA DE CHILE, 2001).

\section{Selección de árboles}

Se utilizaron 40 árboles de Eucalyptus nitens de 13 años, los que presentaron un diámetro a la altura de pecho (DAP) de 43,5 cm y una altura total de 35,26 m. También fueron seleccionados 12 árboles de 15 años, los que presentaron un DAP promedio de $48,5 \mathrm{~cm}$ y una altura total de $37,01 \mathrm{~m}$. Por último, se utilizaron 5 árboles de 20 años, los que presentaron un DAP promedio de $55,5 \mathrm{~cm}$ y una altura total de $40,31 \mathrm{~m}$.

\section{Régimen silvícola}

Los árboles de 13, 15 y 20 años, tuvieron una densidad inicial de 1.666 árboles/ha, con un raleo a los 6 años (700 árboles/ha), a los 8 años (400 árboles/ha) y a los 10 años (200 árboles/ha). Adicionalmente, se les realizó una poda a los 2,5 años (a los 2,5 m de altura), a los 3,5 años (entre los $5 \mathrm{~m}$ y $6 \mathrm{~m}$ de altura) y a los 5 años (a los $9 \mathrm{~m}$ de altura).

\section{Obtención de las muestras}

De cada árbol, se cortaron secciones (rodelas) de $5 \mathrm{~cm}$ de espesor, aplicando el siguiente plan de muestreo; para los árboles de 13 y 20 años, se sacó la primera rodela a los $0,3 \mathrm{~m}$ del suelo (tocón), la segunda a los 1,3 m y luego cada cuatro metros hasta los 9,3 m y 13,3 m, respectivamente. A partir de este punto, se obtuvieron rodelas cada $2,44 \mathrm{~m}$ hasta el Diámetro Límite Utilizable de 8 cm (DLU 8). Para los árboles de 15 años, la primera rodela fue a los $0,3 \mathrm{~m}$ del suelo (tocón), luego dos rodelas cada cuatro metros hasta los $8,3 \mathrm{~m}$ y a partir de este punto se extrajeron rodelas cada 2,44 m, finalizando el muestreo en el DLU 8 (Tabla 1). 
Tabla 1 - Secciones de altura obtenidas en árboles de Eucalyptus nitens de 13, 15 y 20 años

Table 1 - Sections of height obtained in Eucalyptus nitens trees of 13, 15 and 20 years old

\begin{tabular}{ccccccccccccccccccc}
\hline \multirow{2}{*}{$\begin{array}{c}\text { Edad } \\
\text { árbol }\end{array}$} & \multicolumn{1}{c|}{$\mathbf{h}_{\mathbf{1}}$} & $\mathbf{h}_{\mathbf{2}}$ & $\mathbf{h}_{\mathbf{3}}$ & $\mathbf{h}_{\mathbf{4}}$ & $\mathbf{h}_{\mathbf{5}}$ & $\mathbf{h}_{\mathbf{6}}$ & $\mathbf{h}_{\mathbf{7}}$ & $\mathbf{h}_{\mathbf{8}}$ & $\mathbf{h}_{\mathbf{9}}$ & $\mathbf{h}_{\mathbf{1 0}}$ & $\mathbf{h}_{\mathbf{1 1}}$ & $\mathbf{h}_{\mathbf{1 2}}$ & $\mathbf{h}_{\mathbf{1 3}}$ & $\mathbf{h}_{\mathbf{1 4}}$ & $\mathbf{h}_{\mathbf{1 5}}$ & $\mathbf{h}_{\mathbf{1 6}}$ & $\mathbf{h}_{\mathbf{1 7}}$ \\
\hline $\mathbf{1 3}$ & 0,3 & 1,3 & 5,3 & 9,3 & 11,7 & 14,1 & 16,5 & 18,9 & 21,3 & 23,7 & 26,1 & 28,5 & 30,9 & 33,3 & - & - & - \\
$\mathbf{1 5}$ & 0,3 & - & 4,3 & 8,3 & 10,7 & 13,1 & 15,5 & 17,9 & 20,3 & 22,7 & 25,1 & 27,5 & 29,9 & 32,3 & 34,7 & 37,1 & - \\
$\mathbf{2 0}$ & 0,3 & 1,3 & 5,3 & 9,3 & 13,3 & 15,7 & 18,1 & 20,5 & 22,9 & 25,3 & 27,7 & 30,1 & 32,2 & 34,9 & 37,3 & 39,7 & 42,1 \\
\hline
\end{tabular}

Fuente: Autores (2020)

En donde: $h_{j}=$ j-ésima sección de altura obtenida del árbol (m), para j: 1, ...,17.

\section{Obtención de las propiedades físicas}

De cada rodela extraída, se determinó el contenido de humedad y la densidad básica según las recomendaciones de la Norma Chilena NCh 176/1Of.86 (INSTITUTO NACIONAL DE NORMALIZACIÓN, 1984) y de la NCh 176/2Of.86 (INSTITUTO NACIONAL DE NORMALIZACIÓN, 1988), respectivamente.

\section{Obtención del tamaño de la muestra}

El tamaño de la muestra de los árboles a utilizar, fue según las recomendaciones de la NCh 1208 Eof.1976 (INSTITUTO NACIONAL DE NORMALIZACIÓN, 1976). En vista que la densidad de la plantación fue de 200 (árboles/ha), para esta norma corresponde utilizar un tamaño de lote entre 151 y 280 (árboles/ha). A este tamaño de lote se le asignó un nivel de inspección de II y un Acceptable Quality Level (AQL) para un tipo de inspección normal y muestreo simple. Sobre la base de lo anterior, la norma indica que el tamaño mínimo de muestreo correspondió a 32 árboles, el que por motivos prácticos durante la faena forestal fue ampliado a 40 árboles. Por otra parte, para estimar el error asociado al tamaño de la muestra, se utilizó la siguiente fórmula (Ecuación 1).

$\mathrm{e}=\sqrt{\left(\mathrm{z}_{1 / 2}^{2} \times \sigma^{2}\right) / \mathrm{n}}$

en donde: $\mathrm{e}=$ error asociado al tamaño de la muestra $(\mathrm{n}) ; \sigma^{2}=$ varianza poblacional; $\mathrm{Z}_{\mathrm{a} / 2}=$ valor $\mathrm{Z}$

correspondiente al nivel de confianza elegido $(\alpha=0,05)$.

\section{Modelos de predicción para las propiedades físicas}

Los modelos utilizados en esta investigación, basaron su predicción en el efecto de la altura y edad del árbol, así como la relación entre el contenido de humedad con la densidad básica, siendo estos detallados en la Ecuación (2), Ecuación (3) y Ecuación (4).

$\gamma_{i}=\beta_{0}+\beta_{1} \times \tau_{i}+\varepsilon$

$\gamma_{j}=\beta_{0}+\beta_{2} \times \mathrm{h}_{j}+\beta_{3} \times \mathrm{h}_{j}^{2}+\varepsilon$

$\gamma_{i j k}=\beta_{0}+\beta_{1} \times \tau_{i}+\beta_{2} \times \mathrm{h}_{j}+\beta_{3} \times \mathrm{h}_{j}^{2}+\beta_{4} \times \delta_{k}+\varepsilon$

en donde: $\gamma_{i j k}=$ propiedad física determinada de la $i$-ésima propiedad física predictora, $j$-ésima altura del árbol y $k$-ésima edad del árbol; $\tau_{i}=i$-ésima propiedad física utilizada como predictora, donde $i=$ 1: contenido de humedad, $i=2$ : densidad básica; $h_{j}=j$-ésima altura del árbol, para $j: 1, \ldots$, DLU8; $\delta_{k}=$ $k$-ésima edad del árbol, para $i=1: 13$ años, $i=2: 15$ años e $i=3: 20$ años; $\varepsilon=$ error residual del modelo; $\beta_{0}$, $\beta_{1}, \beta_{2}, \beta_{3}$ y $\beta_{4}=$ parámetros del modelo. 


\section{Análisis estadístico}

Se realizó un análisis exploratorio de datos (AED), que permitió identificar datos atípicos, los que mediante un análisis exhaustivo, fueron excluidos del análisis con el fin de mejorar la resolución de los resultados. La estadística descriptiva se aplicó a cada variable analizada, obteniendo las medidas de tendencia central, de dispersión, valores máximos y mínimos e intervalos al 95\% de confianza. Los supuestos de independencia (test de Durbin-Watson), de correlación (test de Pearson) y de normalidad (test de Kolmogórov-Smirnov) fueron verificados. Se desarrolló un análisis de varianza (ANOVA) con un nivel de significancia del 5\% (valor-p: $0,05)$ y la comparación entre medias fue mediante el test de Tukey (valor-p: 0,05). Los modelos estadísticos se confeccionaron con las variables que resultaron significativas, aplicando el procedimiento step wise regression con un nivel de significancia del 5\% (valor-p: 0,05 ), por medio del software estadístico Minitab16.

\section{Resultados y discusión}

\section{Elección del tamaño de la muestra}

Los resultados obtenidos (Tabla 2) mostraron que el número de árboles, necesarios para obtener un error de muestreo del 5\% fue de 120 para la densidad básica y de 27 para el contenido de humedad. Para un error de muestreo del 10\%, fueron necesarios 30 árboles para la densidad básica y 7 árboles para el contenido de humedad. Finalmente, el error de muestreo para un tamaño de muestra correspondiente a los 40 árboles analizados (n: 40), fué de 8,7\% para la densidad básica y $4,1 \%$ para el contenido de humedad.

En términos generales, la densidad básica presentó mayor varianza que el contenido de humedad, por lo que esta última necesitó un tamaño de muestra menor. Basándose en esto, se debe considerar a la densidad básica como la propiedad que restringe la selección del tamaño de muestra. Esto evidencia que, en este tipo de investigación se debe definir de forma adecuada y metodológica el tamaño de muestra a utilizar. Lo anterior es respaldado por Valdivieso, Valdivieso y Valdivieso (2011), quienes recalcan que la decisión del tamaño de la muestra no debe considerarse trivial. Entonces, se debe evitar el uso de reglas intuitivas, sin la adecuada justificación, tales como asignar un tamaño de muestra según la proporción de la población, o asignar un valor excesivamente superior al recomendado, puesto que puede generar errores metodológicos. En vista de lo anterior, Montgomery (1991) sugiere un tamaño de muestra acorde a los objetivos de la investigación recomendando criterios como; la diferencia estadística a obtener, la variación esperada de los datos, el número de tratamientos y factores, la complejidad del análisis estadístico y del tamaño de la población.

Se observa para un mismo error muestral que el contenido de humedad requiere un tamaño de muestra menor en comparación a la densidad básica. Además, si consideramos la relación inversa existente entre ambas propiedades, tal como lo reportaron Chafe (1985), Purnell (1988), Lausberg, Gilchrist y Skipwith (1995), Rozas et al. (2002), Allen et al. (2005), Omonte y Valenzuela (2011), es posible correlacionar ambas propiedades empleando un tamaño de lote sugerido para el contenido de humedad. Este enfoque permitiría reducir la cantidad de mediciones, costos y tiempo de muestreo, sin disminuir la precisión en los valores de ambas propiedades. Sin embargo, la afirmación anterior debería ser corroborada en futuras investigaciones. 
Tabla 2 - Tamaño de muestra y error asociado para densidad básica y contenido de humedad en árboles de Eucalyptus nitens de 13 años

Table 2 - Sample size and associated error for basic density and moisture content in Eucalyptus nitens trees of 13 years old

\begin{tabular}{lcccccc}
\hline & Densidad básica & & & \multicolumn{3}{c}{ Contenido de humedad } \\
\cline { 1 - 4 } \cline { 6 - 7 } Error (\%) & Varianza & $\mathbf{n}$ & & Error $(\mathbf{\%}){ }^{*}$ & Varianza & $\mathbf{n}$ \\
\hline $\mathbf{5 , 0}$ & 752,9 & 120 & & 4,1 & 168,3 & 40 \\
$\mathbf{6 , 0}$ & 752,9 & 83 & & 5,0 & 168,3 & 27 \\
$\mathbf{7 , 0}$ & 752,9 & 61 & & 7,0 & 168,3 & 14 \\
$\mathbf{8 , 7}$ & 752,9 & 40 & & 8,0 & 168,3 & 11 \\
$\mathbf{9 , 0}$ & 752,9 & 37 & & 9,0 & 168,3 & 8 \\
$\mathbf{1 0 , 0}$ & 752,9 & 30 & & 10,0 & 168,3 & 7 \\
\hline
\end{tabular}

Fuente: Autores (2020)

En donde: $\mathrm{n}=$ número de árboles. $\left(^{*}\right)$ Error obtenido con un nivel de confianza del $95 \%(\alpha=0,05)$.

\section{Variación del DAP con la edad de los árboles de Eucalyptus nitens}

La Figura 1 y Figura 2, muestra el histograma de frecuencias e intervalos de confianza al 95\% del DAP agrupado por edad del árbol respectivamente. De ambas Figuras, se observa un aumento del DAP con la edad del árbol, pasando de un DAP de 43,7 cm a los 13 años, a 48,5 cm a los 15 años, lo que representa un aumento del 10,8\%. Para los árboles de 20 años, el DAP fue de $55,5 \mathrm{~cm}$, con un aumento del $26,8 \%$ con respecto al DAP de 13 años. Este incremento del DAP con la edad, fue estadísticamente significativo según los resultados del test HSD de Tukey.

Figura 1 - Histograma de frecuencias del DAP agrupados por edad del árbol

Figure 1 - Histogram corresponding to DBH grouped by tree age

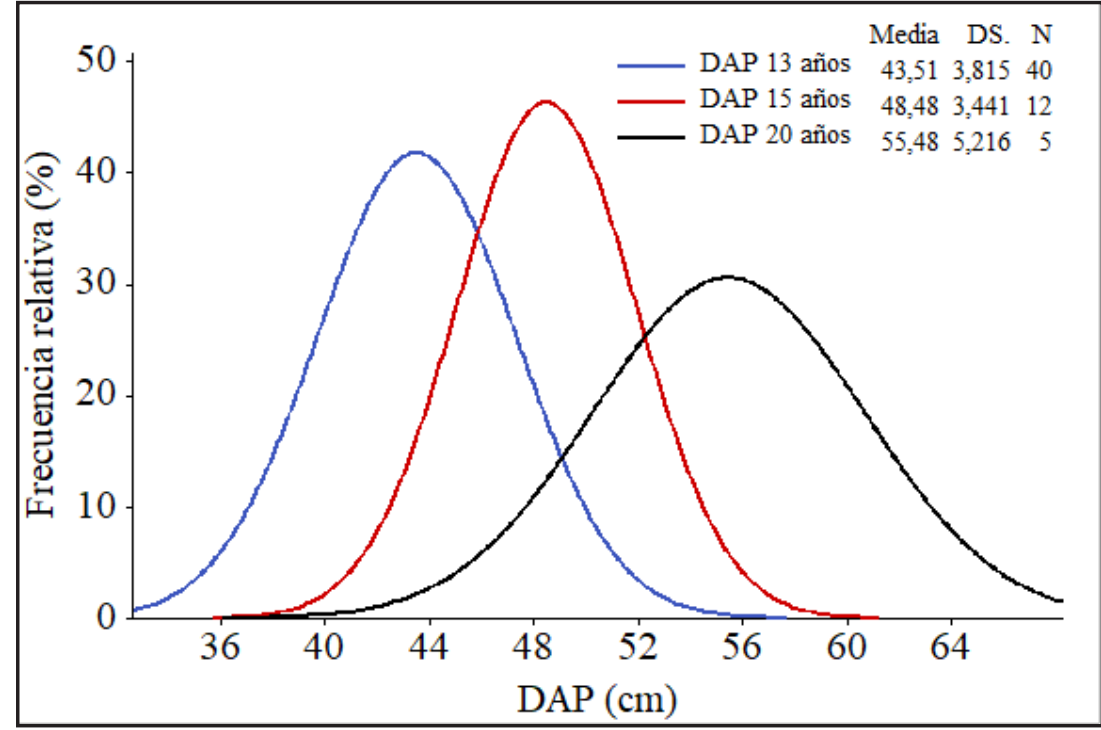

Fuente: Autores (2020) 
Figura 2 - Intervalos de confianza para el DAP en árboles de Eucalyptus nitens de 13, 15 y 20 años

Figure 2 - Confidence intervals for DAP measurements in Eucalyptus nitens trees of 13, 15 and 20 years old

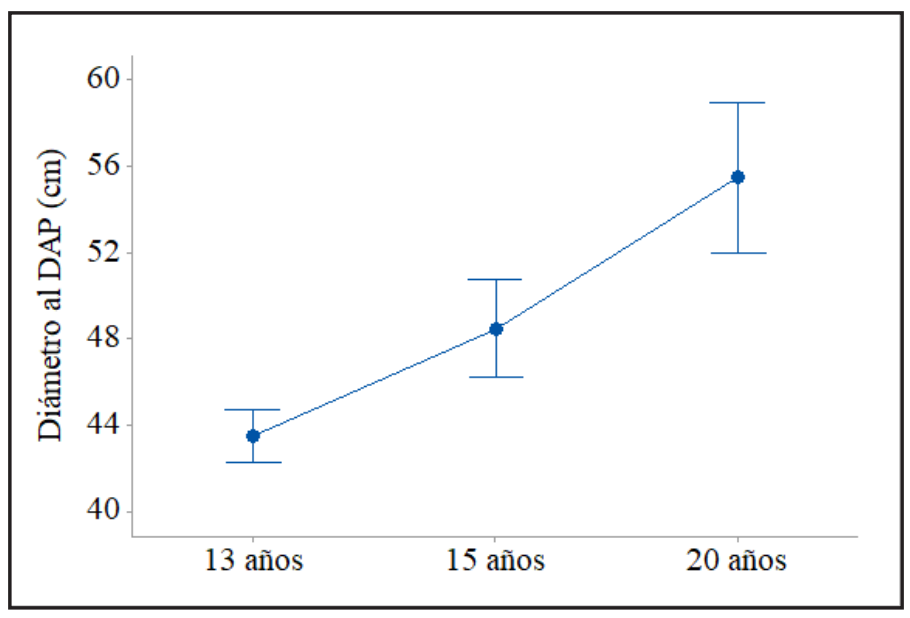

Fuente: Autores (2020)

\section{Variación de la densidad básica con la edad y la altura del árbol}

Se observó un incremento de la densidad básica con la edad del árbol (Tabla 3), siendo la media a los 13 años de $474 \mathrm{~kg} / \mathrm{m}^{3}$, un mínimo de $425 \mathrm{~kg} / \mathrm{m}^{3}$, un máximo de $588 \mathrm{~kg} / \mathrm{m}^{3}$ e intervalo de confianza de 471-479 kg/m³. De modo similar, la media a los 15 años fue de $496 \mathrm{~kg} / \mathrm{m}^{3}$, con un mínimo de $425 \mathrm{~kg} / \mathrm{m}^{3}$, un máximo de $586 \mathrm{~kg} / \mathrm{m}^{3}$ e intervalo de confianza de $491-505 \mathrm{~kg} / \mathrm{m}^{3}$. En cambio, la densidad básica a los 20 años, tuvo una media de $521 \mathrm{~kg} / \mathrm{m}^{3}$, un mínimo de $436 \mathrm{~kg} / \mathrm{m}^{3}$, máximo de 634 $\mathrm{kg} / \mathrm{m}^{3}$ e intervalo de confianza de $508-531 \mathrm{~kg} / \mathrm{m}^{3}$. Estos resultados evidenciaron un aumento relativo del 4,6\% de la densidad básica de 15 años con respecto a los 13 años. En tanto que, a los 20 años este aumento fue de $9,7 \%$ con respecto a los árboles de 13 años.

Por otra parte, tanto el coeficiente de variación (CV) como la desviación estándar (DS), fueron similares para las tres edades estudiadas, mientras que la media e intervalo de confianza (IC) reflejaron el incremento de la densidad básica con la edad en los árboles de Eucalyptus nitens.

En términos generales, la densidad básica fue similar a la reportada por Paz (1993), quien obtuvo valores entre $496 \mathrm{~kg} / \mathrm{m}^{3}$ y $507 \mathrm{~kg} / \mathrm{m}^{3}$ en Eucalyptus nitens de 14 y 16 años respectivamente. De modo similar Rozas et al. (2002), consiguieron valores entre $489 \mathrm{~kg} / \mathrm{m}^{3}$ y $539 \mathrm{~kg} / \mathrm{m}^{3}$ en Eucalyptus nitens de 11 años. Rozas y Lisperguer (2005), reportaron una densidad básica con media entre $496 \mathrm{~kg} / \mathrm{m}^{3}$ y $598 \mathrm{~kg} / \mathrm{m}^{3}$ en Eucalyptus nitens de 11 años. Mariani et al. (2005), informaron una densidad básica entre $450 \mathrm{~kg} / \mathrm{m}^{3}$ y $527 \mathrm{~kg} / \mathrm{m}^{3}$ en Eucalyptus nitens de 9 años, mientras que Gacitúa et al. (2007), comunicaron una densidad básica al DAP entre $469 \mathrm{~kg} / \mathrm{m}^{3}$ y $493 \mathrm{~kg} / \mathrm{m}^{3}$ en Eucalyptus nitens de 16 años. Ananías, Díaz y Leandro (2009), al calcular la densidad básica al DAP indicaron un valor entre $436 \mathrm{~kg} / \mathrm{m}^{3}$ y 536 $\mathrm{kg} / \mathrm{m}^{3}$ en Eucalyptus nitens de 16 años. Poblete y Burgos (2010), alcanzaron una densidad básica de 440 $\mathrm{kg} / \mathrm{m}^{3}$, mientras que Díaz et al. (2012), obtuvieron valores entre $473 \mathrm{~kg} / \mathrm{m}^{3}$ y $492 \mathrm{~kg} / \mathrm{m}^{3}$ en Eucalyptus nitens de 15 años de edad para ambos casos.

Con respecto a la variación de la densidad básica con la altura del árbol, se observó una disminución desde el tocón $(0,3 \mathrm{~m})$ hasta los $5,3 \mathrm{~m}$, presentando en este punto el mínimo valor del árbol. A partir de esta altura, la densidad básica se incrementó de forma lineal hasta el DLU 8, siendo más pronunciado este efecto a partir del último tercio de la altura total, es decir aproximadamente desde los $20 \mathrm{~m}$ (ver Figura 3). La tendencia observada resultó idéntica para las tres edades analizadas, con la salvedad que la densidad básica también aumentó con la edad del árbol para una misma altura. Este patrón de variación, fue reportado por Chafe (1985), en Eucalyptus regnans y por Purnell 
(1988), Lausberg, Gilchrist y Skipwith (1995), Rozas et al. (2002), Rozas y Lisperguer (2005), Leandro et al. (2008) en Eucalyptus nitens, quienes observaron una variación de la densidad básica con la altura, desde los 0,3 m hasta los 30,3 m, con un mínimo a una altura cercana a los $5 \mathrm{~m}$. Navarrete et al. (2008), detectaron tendencia similar, aumentando desde el tocón $\left(484 \mathrm{~kg} / \mathrm{m}^{3}\right)$, hasta los $30,9 \mathrm{~m}\left(549 \mathrm{~kg} / \mathrm{m}^{3}\right)$, con un mínimo cerca del DAP $\left(449 \mathrm{~kg} / \mathrm{m}^{3}\right)$. En general, este comportamiento fue similar para las tres edades analizadas, con la salvedad de que también aumentó con la edad del árbol, lo que según Chafe (1985), se debe al incremento de la edad cambial, la que está estrechamente relacionada con el incremento de la densidad promedio.

Tabla 3 - Estadística descriptiva para la densidad básica $\left(\mathrm{kg} / \mathrm{m}^{3}\right)$ y contenido de humedad (\%) en árboles de Eucalyptus nitens de 13, 15 y 20 años

Table 3 - Descriptive statistics of basic density $\left(\mathrm{kg} / \mathrm{m}^{3}\right)$ and moisture content (\%) for Eucalyptus nitens trees of 13, 15 and 20 years old

\begin{tabular}{lccccc}
\hline Propiedad física & Estadístico & Unidad & $\mathbf{1 3}$ años & $\mathbf{1 5}$ años & 20 años \\
\hline \multirow{2}{*}{ Densidad básica } & $\mathrm{N}$ & & 412 & 153 & 78 \\
& Media & $\mathrm{kg} / \mathrm{m}^{3}$ & 474 & 496 & 521 \\
& $\mathrm{DS}$ & $\mathrm{kg} / \mathrm{m}^{3}$ & 44 & 45 & 53 \\
& $\mathrm{CV}$ & $\%$ & 9,2 & 9,0 & 10,2 \\
& Mín & $\mathrm{kg} / \mathrm{m}^{3}$ & 425 & 425 & 436 \\
& Máx & $\mathrm{kg} / \mathrm{m}^{3}$ & 588 & 586 & 634 \\
& IC & $\mathrm{kg} / \mathrm{m}^{3}$ & $471-479$ & $491-505$ & $508-531$ \\
\hline \multirow{3}{*}{ Contenido de humedad } & $\mathrm{n}$ & 421 & 151 & 70 \\
& Media & $\%$ & 116 & 108 & 104 \\
& DS. & $\%$ & 20 & 20 & 18 \\
& MV & $\%$ & 17,5 & 18,3 & 17,4 \\
& Mín & $\%$ & 70 & 71 & 74 \\
& Máx & $\%$ & 159 & 147 & 137
\end{tabular}

Fuente: Autores (2020)

En donde: $\mathrm{n}=$ número de mediciones efectuadas, Media = Valor medio de la muestra, $\mathrm{DS}=$ Desviación estándar, $\mathrm{CV}=$ Coeficiente de variación, Min = Valor mínimo de la muestra, Max = Valor máximo de la muestra, IC = Intervalo de confianza, con nivel de confianza del 95\%.

Este comportamiento, también se detectó en otras especies de Eucalyptus tal como lo expusieron Labbé et al. (2013), en árboles de Eucalyptus globulus y Omonte y Valenzuela (2011) en árboles de Eucalyptus regnans. En principio, las causas de la variación de la densidad básica con la altura del árbol no están bien definidas, Zobel y Van Buijtenen (1989), lo atribuyen a la formación de madera de reacción y a factores de crecimiento. También, a la relación entre la madera inicial y tardía (LEANDRO et al., 2008). Con respecto a este comportamiento, Igartúa et al. (2002), Rocha et al. (2019), evidenciaron que los Eucalyptus aumentan su densidad con el incremento de la altura del árbol, presentando en algunos casos una disminución cerca del DAP. Esta variabilidad se debe según Malan y Hoon (1992) y Rocha et al. (2019), a la proporción entre la madera adulta y madera juvenil. En este sentido, existe amplio consenso en los efectos negativos de la madera juvenil sobre las propiedades físicas y mecánicas de la madera, la cual se relaciona con una menor densidad y resistencia mecánica, 
fibras de menor longitud y un elevado ángulo microfibrilar en comparación a la madera madura, los que reducen la calidad y el rendimiento productivo de la madera procesada.

\section{Variación del contenido de humedad con la edad y la altura del árbol}

Los resultados del contenido de humedad (Tabla 3), presentaron a los 13 años una media del $116 \%$, un mínimo de $70 \%$, máximo de $159 \%$ e intervalo de confianza de $115 \%-118 \%$. A los 15 años, la media fue de $108 \%$, un mínimo de $71 \%$, un máximo de $147 \%$ e intervalo de confianza de $105 \%-111 \%$. Finalmente, a los 20 años la media fue de 104\%, un mínimo de 74\%, máximo de $104 \%$ e intervalo de confianza de 100\%-108\%. Además, estos resultados mostraron un coeficiente de variación (CV) y desviación estándar (DS), similares para las tres edades. También se observó una disminución del 6,9\% entre las mediciones efectuadas a los 15 años con respecto a las de 13 años. En tanto que a los 20 años esta disminución fue del 10,3\%.

Estos resultados fueron similares a los reportados por Lausberg, Gilchrist y Skipwith (1995), quienes obtuvieron un contenido de humedad entre 106\%-162\% en Eucalyptus nitens de 15 años. Por su parte, Rozas, Sánchez y Pinedo (2005), registraron valores entre $117 \%-137 \%$ en piezas de Eucalyptus nitens recién aserradas y por Allen et al. (2005), quienes reportaron valores medios de 155\%, 104\% y $97 \%$ en Eucalyptus nitens de 5, 8 y 12 años, respectivamente.

La variación del contenido de humedad con la altura (Figura 3), mostró un aumento desde los 0,3 m hasta cerca del DAP en árboles de 13 y 20 años y hasta $4,3 \mathrm{~m}$ en árboles de 15 años, siendo a esa altura el contenido de humedad máximo. A partir de esta, se observó una disminución sostenida hasta el DLU 8, mostrando desde los $30 \mathrm{~m}$, una pendiente menor a la observada a alturas inferiores, lo que evidenció la no linealidad del incremento. De modo similar, se detectó una disminución del contenido de humedad con el aumento de la edad, lo cual se atribuye al aumento de la densidad básica con la edad del árbol. Es relevante indicar que, esta variación, también fue advertida por Chafe (1985), Purnell (1988), Lausberg, Gilchrist y Skipwith (1995), Rozas et al. (2002), Allen et al. (2005), Omonte y Valenzuela (2011), quienes lo atribuyen a la relación inversa entre el contenido de humedad y la densidad básica, lo que para esta investigación resultó ser válido para todas las alturas y edades estudiadas.

Figura 3 - Variación de la densidad básica (DB) $\left(\mathrm{kg} / \mathrm{m}^{3}\right)$ y contenido de humedad $(\mathrm{CH})(\%)$ con la altura y edad en árboles de Eucalyptus nitens

Figure 3 - Variation of basic density (DB) $\left(\mathrm{kg} / \mathrm{m}^{3}\right)$ and moisture content $(\mathrm{CH})(\%)$ at different heights and age in Eucalyptus nitens trees

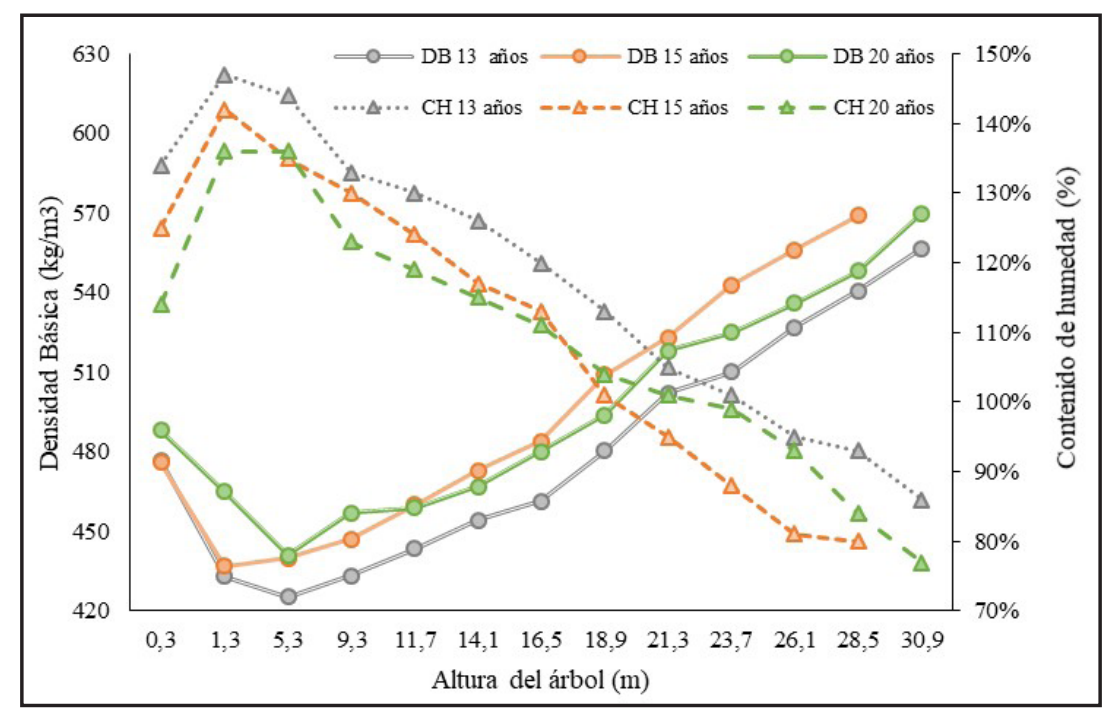

Fuente: Autores (2020) 


\section{Correlación entre la densidad básica y el contenido de humedad}

Las evidencias descritas anteriormente, muestran un grado de relación entre la densidad básica y el contenido de humedad. Esto fue validado mediante un análisis de correlación de Pearson, el cual mostró una elevada relación inversa, siendo esta estadísticamente significativa (valor-p: <0,001), evidenciando de esta forma un comportamiento inversamente proporcional entre ambas (Tabla 4). Debido a lo anterior, se ajustó un modelo lineal y otro de segundo orden, que permitió predecir a la densidad básica y contenido de humedad en árboles de Eucalyptus nitens de 13, 15 y 20 años y a diferentes alturas. La Tabla 5, resume los parámetros y estadísticos de cada modelo, observando que estos fueron significativos (valor-p: $<0,001$ ) y que presentaron un elevado coeficiente de determinación ( $\mathrm{r}^{2}$ entre 0,7124 y 0,9211$)$.

Con respecto a este comportamiento, Oliveira y Silva (2003) y Derikvand et al. (2019), indicaron que la variación de la densidad, repercute sobre el contenido de humedad. Esto se explica desde el punto de vista anatómico por la proporción de vasos y espesor de las paredes celulares de las fibras. De esta forma, una mayor densidad, puede ocurrir a causa del incremento del espesor de la pared celular, o bien por el aumento en la cantidad de fibras en relación a la proporción de vasos. Mientras que una mayor proporción de vasos, independiente de la variación del espesor de la pared celular, ocasionará una menor densidad (OLIVEIRA; SILVA, 2003; ROCHA et al., 2019). En general, la correlación entre ambas propiedades físicas, concuerda con lo indicado por Chafe (1985), en árboles de Eucalyptus regnans y por Purnell (1988) en árboles de Eucalyptus nitens, quienes mencionan la existencia de una relación inversa y altamente significativa entre ambas propiedades. De ese modo, debido a la naturaleza del comportamiento de la densidad básica y del contenido de humedad con la altura, el modelo que tuvo mejores resultados, fue el que consideró el término de segundo orden de la altura del árbol, entregando resultados aceptables para las tres edades estudiadas. Este tipo de modelos de regresión, también fue propuesto por Lausberg, Gilchrist y Skipwith (1995), Navarrete et al. (2008), Omonte y Valenzuela (2011), Labbé et al. (2013), en otras especies de Eucalyptus, los que también presentaron una relación significativa entre estas dos propiedades físicas.

Tabla 4 - Matriz de correlación de Pearson entre densidad básica y contenido de humedad en árboles de Eucalyptus nitens de 13, 15 y 20 años

Table 4 - Pearson correlation coefficient between the basic density and moisture content in Eucalyptus nitens trees of 13, 15 and 15 years old

\begin{tabular}{lcccc}
\hline \multirow{2}{*}{ Densidad básica } & & \multicolumn{3}{c}{ Contenido de humedad } \\
\cline { 3 - 4 } & & $\mathbf{1 3}$ años & $\mathbf{1 5}$ años & $\mathbf{2 0}$ años \\
\hline \multirow{2}{*}{$\mathbf{1 3}$ años } & CP & $-0,844$ & \\
\multirow{2}{*}{$\mathbf{1 5}$ años } & Valor-p & $<0,001^{*}$ & & \\
& $\mathrm{CP}$ & & $-0,896$ & \\
\multirow{2}{*}{$\mathbf{2 0}$ años } & Valor-p & $<0,001^{*}$ & $-0,866$ \\
& $\mathrm{CP}$ & & $<0,001^{*}$ \\
\hline
\end{tabular}

Fuente: Autores (2020)

En donde: $\mathrm{CP}=$ valor coeficiente de correlación de Pearson con nivel de confianza del 95\%, Valor-p = probabilidad, (*) Significativo a un nivel de 5\% de probabilidad de error, por coeficiente de correlación de Pearson. 
Tabla 5 - Parámetros y estadísticos del modelo $\gamma_{\mathrm{i}}=\boldsymbol{\beta}_{0}+\boldsymbol{\beta}_{1} \times \tau_{\mathrm{i}}+\varepsilon$ y del modelo $\gamma_{\mathrm{j}}=\boldsymbol{\beta}_{0}+\boldsymbol{\beta}_{2} \times \mathbf{h}_{\mathrm{j}}+\boldsymbol{\beta}_{3} \times \mathbf{h}_{\mathrm{j}}{ }^{2}+\varepsilon$, para la predicción de propiedades físicas

Table 5 - Parameters and statistics of the model $\gamma_{i}=\beta_{0}+\beta_{1} \times \tau_{i}+\varepsilon$ and model $\gamma_{j}=\beta_{0}+\beta_{2} \times h_{j}+\beta_{3} \times h_{j}{ }^{2}+\varepsilon$, for the prediction of physical properties

\begin{tabular}{|c|c|c|c|c|c|c|c|c|}
\hline \multirow{2}{*}{$\begin{array}{c}\text { Propiedad física } \\
\text { evaluada }\left(\gamma_{\mathrm{ij}}\right)\end{array}$} & \multirow{2}{*}{$\begin{array}{c}\text { Edad árbol } \\
\text { (años) }\end{array}$} & \multirow{2}{*}{ Unidad } & \multicolumn{4}{|c|}{ Parámetros } & \multicolumn{2}{|c|}{ Estadísticos } \\
\hline & & & $\beta_{0}$ & $\beta_{1}$ & $\boldsymbol{\beta}_{2}$ & $\boldsymbol{\beta}_{3}$ & $\mathbf{r}^{2}$ & valor-p \\
\hline \multirow{6}{*}{$\begin{array}{l}\text { Densidad } \\
\text { básica }\end{array}$} & 13 & $\mathrm{~kg} / \mathrm{m}^{3}$ & 683,7 & $-1,745$ & 0 & 0 & 0,7124 & $<0,001^{*}$ \\
\hline & 15 & $\mathrm{~kg} / \mathrm{m}^{3}$ & 706,7 & $-1,919$ & 0 & 0 & 0,8023 & $<0,001^{*}$ \\
\hline & 20 & $\mathrm{~kg} / \mathrm{m}^{3}$ & 768,4 & $-2,240$ & 0 & 0 & 0,7504 & $<0,001^{*}$ \\
\hline & 13 & $\mathrm{~kg} / \mathrm{m}^{3}$ & 444,1 & 0 & $-2,439$ & 0,211 & 0,7622 & $<0,001^{*}$ \\
\hline & 15 & $\mathrm{~kg} / \mathrm{m}^{3}$ & 441,3 & 0 & $-1,005$ & 0,335 & 0,9211 & $<0,001^{*}$ \\
\hline & 20 & $\mathrm{~kg} / \mathrm{m}^{3}$ & 461,5 & 0 & $-0,128$ & 0,206 & 0,9007 & $<0,001^{*}$ \\
\hline \multirow{6}{*}{$\begin{array}{l}\text { Contenido } \\
\text { de } \\
\text { humedad }\end{array}$} & 13 & $\%$ & 313,4 & $-4,082$ & 0 & 0 & 0,7124 & $<0,001^{*}$ \\
\hline & 15 & $\%$ & 316,9 & $-4,180$ & 0 & 0 & 0,8023 & $<0,001^{*}$ \\
\hline & 20 & $\%$ & 266,2 & $-3,128$ & 0 & 0 & 0,7504 & $<0,001^{*}$ \\
\hline & 13 & $\%$ & 143,2 & 0 & 1,178 & 0,024 & 0,7997 & $<0,001^{*}$ \\
\hline & 15 & $\%$ & 138,2 & 0 & 1,041 & 0,022 & 0,8552 & $<0,001^{*}$ \\
\hline & 20 & $\%$ & 133,7 & 0 & 1,089 & 0,098 & 0,9030 & $<0,001^{*}$ \\
\hline
\end{tabular}

Fuente: Autores (2020)

En donde: $\gamma_{\mathrm{ij}}=$ propiedad física determinada de la i-ésima propiedad física predictora y $\mathrm{j}$-ésima altura del árbol; $\mathrm{h}_{\mathrm{j}}=$ j-ésima altura del árbol (m), para j: 1, ..., DLU8; $\tau_{\mathrm{i}}=\mathrm{i}$-ésima propiedad física, donde $\mathrm{i}=1$ : contenido de humedad, $\mathrm{i}=2$ : densidad básica; $\beta_{0}, \beta_{1}, \beta_{2}$ y $\beta_{3}$ = Parámetros del modelo; $\mathrm{r}^{2}$ = Coeficiente de determinación; valor- $\mathrm{p}=$ Probabilidad; *Significativo a un nivel de significancia del $5 \%$, mediante prueba $\mathrm{F}$.

\section{Modelo general para la estimación de la densidad básica}

Envista del impacto de la densidad básica sobre la mayoría de las propiedades físicas y mecánicas de la madera, se aplicó un análisis de varianza (ANOVA) sobre esta propiedad, el cual mostró que los factores significativos, fueron el término de primer orden del contenido de humedad, de la altura y edad del árbol y el término de segundo orden de la altura del árbol. Mediante la prueba-F, se obtuvo que el término de segundo orden de la altura, tuvo el mayor impacto sobre la densidad básica, lo cual se relaciona con el incremento no lineal de la densidad básica con la altura del árbol. En segundo orden de importancia, se encontró el término de primer orden del contenido de humedad, mientras que el factor edad del árbol a pesar de ser significativo, no tuvo gran efecto sobre la densidad básica.

Finalmente, el modelo presentó un valor-F de 492,77, que resultó ser altamente significativo (valor-p: $<0,001$ ), por lo que el modelo tiene una probabilidad menor al $0,01 \%$, de haber sido afectado por otro factor no incluido en la investigación. Por otra parte, el coeficiente de determinación $\left(\mathrm{r}^{2}\right)$ fue de 0,7393 , lo que permitiría afirmar que el modelo, puede predecir adecuadamente la densidad básica, considerando como predictores las variables significativas mencionadas anteriormente, siendo sus parámetros y factores descritos en la Ecuación 5.

$$
\gamma_{i j k}=627,6-1,334 \times C H_{i}-3,039 \times h_{j}+0,133 \times h_{j}^{2}+0,822 \times \delta_{k}
$$

en donde: $\gamma_{\mathrm{ijk}}=$ densidad básica $\left(\mathrm{kg} / \mathrm{m}^{3}\right)$ obtenida al i-ésimo contenido de humedad, la j-ésima altura, y la k-ésima edad del árbol; $h_{\mathrm{i}}=\mathrm{j}$-ésima altura del árbol $(\mathrm{m})$, para j: 1, ..., DLU8; $\mathrm{CH}_{\mathrm{i}}=\mathrm{i}$-ésimo contenido de humedad (\%), i: 68, ...,188; $\delta_{\mathrm{k}}=$ k-ésima edad del árbol,parai=1: 13 años, i=2: 15 años e i=3: 20 años. 
En general, este tipo de modelo se ha usado para predecir las propiedades físicas en otras especies con diversos propósitos, tal como lo mencionaron Busnardo et al. (1987), quienes modelaron matemáticamente las diferentes variables del árbol, para detectar la altura óptima en la que es posible obtener una densidad básica representativa en Eucalyptus regnans, empleando solo una medición. Shelbourne et al. (2002) utilizaron un modelo similar para evaluar el efecto del sitio, condiciones climáticas y edad del árbol sobre la densidad en Eucalyptus nitens. Sette et al. (2012), evaluaron mediante este método la variación de la densidad con la altura y edad en árboles de Eucalyptus grandis. Mientras que Gutiérrez-Vásquez et al. (2012), los aplicaron para modelar la variación de la densidad en árboles de Cedrela odorata L. En tanto Beets et al. (2018), propusieron un modelo para predecir la variación de la densidad en árboles de Douglas Fir, empleando como predictores a factores climáticos y de sitio. Omonte et al. (2019), los emplearon para estimar la densidad verde en Eucalyptus nitens.

Desde un punto de vista práctico, la metodología y los modelos propuestos por esta investigación se pueden aplicar para predecir la densidad básica, lo cual permitiría apoyar la correcta selección de árboles de Eucalyptus nitens, así como definir el adecuado uso productivo de trozas de esta especie para obtención de madera aserrada, tableros o celulosa donde se emplea como criterio de calidad a la densidad básica, la cual presenta variación significativa con altura y edad del árbol.

\section{Conclusiones}

Los árboles de Eucalyptus nitens seleccionados, presentaron una correlación inversa significativa, entre la densidad básica y contenido de humedad en las edades y alturas estudiadas.

Se detectó una variación de la densidad básica con la altura del árbol, la cual disminuyó desde los 0,3 m al DAP y luego se incrementó hasta el DLU 8. En tanto que, el contenido de humedad tuvo el comportamiento inverso para las condiciones evaluadas.

Los modelos de primer y segundo orden para predecir ambas propiedades físicas, fueron altamente significativos (valor-p: <0,001) y mostraron adecuados coeficientes de determinación. De igual modo, el modelo general de regresión múltiple, fue altamente significativo y con alta capacidad de predicción, siendo la altura y contenido de humedad, las de mayor poder predictivo mientras que la edad tuvo un menor efecto a pesar de ser estadísticamente significativo.

En vista de los resultados, tanto la metodología como los modelos propuestos pueden emplearse para caracterizar estas propiedades físicas, con el fin de definir la utilización de trozas de esta especie para madera sólida, tableros o celulosa. Finalmente, se recomienda ampliar estos resultados para otros sitios y condiciones climáticas, con el fin de verificar los patrones de comportamiento detectados en esta investigación.

\section{Agradecimientos}

Los autores agradecen al Sr. Michael Bregar, Gerente General de la empresa Agrícola y Forestal Natalhue Ltda. por el aporte de los árboles necesarios para realizar este estudio. Al fondo de Desarrollo Científico y Tecnológico proyecto Fondef Idea $\mathrm{N}^{\circ} \mathrm{CA} 12 \mathrm{I} 10289$, al Proyecto CD-INES 15-02 y al Proyecto CD-INES 15-14 por el apoyo financiero. 


\section{Referencias}

ALLEN, J. E. et al. Fabricación de OSB y contrachapado a partir de Eucalyptus nitens: análisis del comportamiento en proceso. 2013. Tese (Ingeniería Civil en Industrias Forestales) - Universidad del Bío-Bío, Concepción, 2005.

ANANÍAS, R.; DÍAZ, C.; LEANDRO, L. Estudio preliminar de la contracción y el colapso en Eucalyptus nitens. Maderas: Ciencia y Tecnología, Concepción, v. 13, n. 3, 251-262, nov. 2009.

BEETS, P. et al.Predicting Wood density of growth increments of Douglas-Fir stands in New Zealand. New Zealand Journal of Forestry Science, Christchurch, v. 48, n. 8, p. 8, dec. 2018.

BURGOS, R. Estudio preliminar sobre el efecto de la inmersión en agua sobre la liberación de las tensiones de crecimiento en trozas de Eucalyptus nitens. 2009. Tese (Ingeniería Civil en Industrias Forestales) - Universidad del Bío-Bío, Concepción, 2009.

BUSNARDO, C. et al. Em busca da qualidade ideal da madeira do eucalipto para produção de celulose. IV. Altura ideal de amostragem para avaliação da densidade media para arvore de E. grandis. In: CONGRESSO ANUAL DA ABCP, 11., 1987, São Paulo. Anais [...]. São Paulo: Associação Brasileira de Celulosa e Papel, 1987. p. 17-33.

CAMPOS, A. et al. Propiedades y usos de especies madereras de corta rotación. Santiago de Chile: Instituto Forestal; Ministerio de Agricultura de Chile, 1990. 119 p. (Informe técnico, 122).

CHAFE, S. C. The distribution and interrelationship of collapse, volumetric shrinkage, moisture content and density in trees of Eucalyptus regnans F. Muell. Wood Science and Technology, München, v. 19, n. 4, p. 329-345, dez. 1985.

DERIKVAND, M. et al. Characterisation of Physical and Mechanical Properties of Unthinned and Unpruned Plantation-Grown Eucalyptus nitens H. Deane \& Maiden Lumber. Forests, [s. l.], v. 10, n. 2, p. 194-208, feb. 2019.

DIRECCIÓN METEOROLÓGICA DE CHILE. Climatología regional. Santiago de Chile, 2001. 45 p. disponible en: http://164.77.222.61/climatologia/

DÍAZ, S. et al. Efecto del raleo en el crecimiento y algunas propiedades de la madera de Eucalyptus nitens en una plantación de 15 años. Maderas. Ciencia y Tecnología, Concepción, v. 14, n. 3, p. 379-388, sep. 2012.

GACITÚA, W. A. et al. Nanoindentaciones y ultra estructura en madera de Eucalyptus nitens con micro y meso grietas. Maderas. Ciencia y Tecnología, Concepción, v. 9, n. 3, p. 259-270, dez. 2007.

GUTIERREZ-VAZQUEZ, B. et al. Variación y predicción de la densidad básica de la madera de Cedrela odorata L. Revista Fitotecnia Mexicana, [s. l.], v. 35, n. 5, sep. 2012.

HILLIS, W.; BROWN, A. Eucalypts for Wood production. Sydney: Academic press, 1984. 434 p.

IGARTÚA, D. V. et al. Calidad del leño en Eucalyptus globulus ssp. globulus. Variaciones de la densidad básica y la longitud de fibras en Loberia, provincia de Buenos Aires, Argentina. Revista de la Facultad de Agronomía, La Plata. v. 105, n. 1, p. 29-39. 2002.

INSTITUTO FORESTAL. Datos Tecnológicos del Eucalyptus nitens. Santiago de Chile, 2010. 4 p.

INSTITUTO FORESTAL. El sector forestal chileno 2016. Santiago de Chile, 2016. 52 p.

INSTITUTO NACIONAL DE NORMALIZACION. NCh 176/1.0f84. Madera. Parte 1: Determinación de humedad. Santiago de Chile, 1984. 13 p.

INSTITUTO NACIONAL DE NORMALIZACION. NCh 176/2.0f86.Madera. Parte 2: 
Determinación de la densidad. Santiago de Chile, 1988. 17 p.

INSTITUTO NACIONAL DE NORMALIZACION. NCh 1208 Eof1976. Control de calidad inspección por variables - Tablas y procedimientos de muestreo. Santiago de Chile, 1976. 107 p.

KIMBERLEY, M. O. et al. Modelling the variation in wood density of New Zealand - grown Douglas fir. New Zealand Journal of Forestry Science, Christchurch, v. 47, n. 15, aug. 2017.

LABBÉ, R. et al. Variación de la densidad de la madera y rendimiento pulpable en clones de Eucalyptus globulus evaluado con espectroscopia de infrarrojo cercano. Bosque, Valdivia, v. 34, n. 3, p. 263-272, jul. 2013.

LAUSBERG, M. J. F.; GILCHRIST, K. F.; SKIPWITH, J. H. Wood properties of Eucalyptus nitens grown in New Zealand. New Zealand Journal of Forestry Science, Christchurch, v. 25, n. 2, p. 147-163, jun. 1995.

LEANDRO, L. et al. Estudio preliminar de las grietas internas en anillos de madera inicial y su relación con características de la estructura anatómica y densidad en Eucalyptus nitens. Interciencia, Caracas, v. 33, n. 11, p. 829-834, nov. 2008.

MALAN, F. S.; HOON, M. Effect of initial spacing and thinning on some wood properties of Eucalyptus grandis. South African Forestry Journal, Pretoria, v. 163, n. 1, p. 13-20, 1992.

MARIANI, S. et al. Caracterización física y química del Eucalyptus nitens con la altura. In: COLOQUIO INTERNACIONAL SOBRE CELULOSA DE EUCALIPTO, 5., Concepción. Anais [...]. Concepción: Asociación Técnica de Celulosa y Papel ATCP, 2005. 7 p.

MONTGOMERY, D. C. Diseño y Análisis de Experimentos. 2. ed. Ciudad de México: Grupo Editorial Iberoamérica, 1991. 692 p.

NAVARRETE, E. et al. Efecto del manejo silvícola y clase de copa sobre la densidad básica de Eucalyptus nitens. Floresta, Curitiba, v. 39, n. 2, n. 345-354, sep. 2008.

OLIVEIRA, J. T. S.; SILVA, J. C. Variação radial de retratibilidade e densidade básica de madeira de Eucalyptus saligna Sm. Revista Árvore, Viçosa, MG, v. 27, n. 3, p. 381-385, 2003.

OMONTE, M. et al. Variación del contenido de humedad verde y de la densidad básica de la madera en árboles de Eucalyptus nitens con dimensiones aserrables. Maderas. Ciencia y Tecnología, Concepción, v. 21, n. 3, p. 413-424, jul. 2019.

OMONTE, M.; VALENZUELA, L. Variación radial y longitudinal de la densidad básica en árboles de Eucalyptus regnans de 16 años. Maderas. Ciencia y Tecnología, Concepción, v. 13, n. 2, p. 211-224, apr. 2011.

PAZ, J. Características físicas, químicas y biométricas de distintas especies de Eucalyptus y su aptitud pulpable. In: ACTAS SIMPOSIO LOS EUCALYPTUS EN EL DESARROLLO FORESTAL EN CHILE, 11., 1993, Pucón. Anais [...]. Pucón: Instituto Forestal, 1993. p. 531-561.

PANSHIN, A.; DE ZEEUW, C. Textbook of wood technology. 4th ed. New York: McGraw-Hill, 1980. $722 \mathrm{p}$.

POBLETE, F.; BURGOS, R. Eucalyptus nitens como materia prima para tableros de partículas. Maderas. Ciencia y Tecnología,Concepción, v. 12, n. 1, p. 25-35, mar. 2010.

PURNELL, R. C. Variation in wood properties of Eucalyptus nitens in a provenance trial on the eastern Transvaal highveld in South Africa. South African Forestry Journal, Pretoria, v. 144, n. 1, p. 10-22, 1988.

QUILHÓ, T.; MIRANDA, I.; PEREIRA, H. Within - tree variation in wood fibre biometry and basic density of the urograndis Eucalypt hybrid (Eucalyptus grandis $\times$ Eucalyptus urophylla). IAWA jornal, Netherlands, v. 27, n. 3, p. 243-254. 2006. 
ROCHA, M. F. V. et al. Do the growing conditions of trees influence the wood properties? Floresta e Ambiente, Seropédica, v. 26, n. 3, p. 16, 2019.

ROZAS, C. et al. Diversificación y aprovechamiento de las plantaciones de Eucalyptus globulus y Eucalyptus nitens para su utilización en la industria del mueble. Concepción: Universidad del Bío-Bío, 2002. 108 p. (Resultados Tecnológicos Proyecto Fondef).

ROZAS, C.; LISPERGUER, J. Paneles unidos de canto y contrachapados fabricados con madera juvenil de Eucalyptus nitens. Bosque, Valdivia, v. 7, n. 3, p. 75-79, apr. 2005.

ROZAS, C.; SÁNCHEZ, R.; PINEDO, P. Drying of Eucalyptus nitens and Eucalyptus globulus for block, furniture and flooring. Maderas. Ciencia y Tecnología, Concepción, v. 7, n. 2, p. 109-120, aug. 2005.

SEDLAR, T. et al. Physical properties of Wood in white poplar clone "L-12" grown in Republic of Croatia and Serbia. Topola, [s. l.], v. 203, nesp, p. 45-51, 2019.

SETTE, C. R. et al. Efeito da idade e posição de amostragem na densidade e características anatômicas da madeira de Eucalyptus grandis. Revista Árvore, Viçosa, MG, v. 36, n. 6, p. 11831190, dez. 2012.

SHELBOURNE, C. J. et al. Wood density and internal checking of young Eucalyptus nitens in New Zealand as affected by site and height up the tree. New Zealand Journal of Forestry Science, Christchurch v. 32, n. 3, p. 357-385, 2002.

TOMAZELLO, M. Variação radial da densidade básica e da estrutura anatômica da madeira do Eucalyptus saligna, e Eucalyptus grandis. Scientia Florestalis, Piracicaba, v. 29, p. 39-45, ago. 1985.

VALDIVIESO, C.; VALDIVIESO, O.; VALDIVIESO, R. Determinación del tamaño muestral mediante el uso de árboles de decisión (No. 0311). Investigación y Desarrollo, La Paz, v. 11, p. 148-176, jan. 2011.

WIMMER, R.; DOWNES, G.; EVANS, R. High-resolution analysis of radial growth and wood density in Eucalyptus nitens, grown under different irrigation regimes. Annals of Forest Science, [s. l.],v. 59, p. 519-524, jul./oct. 2002.

ZOBEL, B.; VAN BURJTENEN, J. Wood variation: its causes and control. New York: Springer Verlag, 1989. $480 \mathrm{p}$. 